\title{
Enhanced energy detection receiver for OOK signals using Gaussian approximation
}

\author{
Dazhong $\mathrm{Mu}^{\mathrm{a}}$, Peng Cao, Huaqun Liu \\ Beijing Key Laboratory of Signal and Information Processing for High-end Printing Equipments, \\ Beijing Institute of Graphic Communication, Beijing, China \\ adazhongmu@bigc.edu.cn
}

Keywords: Energy detector, UWB communication, OOK signal

\begin{abstract}
Equal-gain combining (EGC) is a conventional linear combining scheme used in non-coherent energy detection receiver for ultra-wideband (UWB) signals. However, due to the nonlinear nature of decision boundary, the EGC results in a suboptimal test statistic. In this paper, we propose a new design for a nonlinear combining energy detector based on Gaussian approximation, in which the energy detection problem becomes a general Gaussian signal detection problem with unequal-mean and unequal-variance. Numerical results indicate that the proposed detector exhibits better performance than the conventional energy detector.
\end{abstract}

\section{Introduction}

Impulse radio (IR) UWB has been considered as a promising solution for supporting low-cost and low-data-rate wireless communication. The energy detection receivers are favored for their simple implementation and good performance-complexity tradeoffs for UWB systems. The conventional energy detection receiver is known as the EGC (square-law combining) receiver, which recovers the transmitted symbol by comparing the combined output to a decision threshold [1,2]. However, it was shown in [3] that EGC for conventional energy detector is not optimal unless the signal-to-noise ratio (SNR) is infinitesimally small.

Since the decision boundary is nonlinear [3], the implementation of optimal energy detector involves nonlinear functional operation, which not only complicates the detector structure, but also makes the exact evaluation of the average BER difficult to obtain analytically. In this letter, we propose a new design for a suboptimal energy detector based on Gaussian approximation. The goal of the suboptimal detector design is to reduce the complexity while preserving the performance very close to the optimal detector. Numerical results using the IEEE 802.15.4a channel models show that the proposed detector can effectively improve bit error rate (BER) performance without significantly increase of the system complexity, as compared to the conventional energy detector.

\section{System model}

Let the received UWB signal after the Rx antenna can be represented as

$r(t)=\sqrt{E_{p}} \sum_{i=-\infty}^{+\infty} \sum_{j=1}^{N_{f}} d_{i} s\left(t-j T_{f}-i T_{s}\right)+n(t)$

Where the frame index and pulse repetition time are denoted by $j$ and $T_{f}, N_{f}$ represents the number of the pulses transmitted per data symbol, $T_{s}$ is the symbol duration, $E_{p}$ is the energy transmitted for each pulse, and $d_{i} \in\{0,1\}$ is the ith transmitted bit with on-off keying (OOK) modulation. The term $s(t)$ is the convolution of the energy-normalized waveform of the transmitted pulse and the normalized impulse response of the UWB radio channel. The ambient noise $n(t)$ is approximated as a zero-mean white Gaussian process with two-sided power spectral density $N_{0} / 2$. 
After the usage of the square-law device as well as integration time over $T_{\text {int }}$, the energy samples of the $i$ th transmitted bit can be achieved as the

$$
y_{i, j}=\int_{i T_{S}+(j-1) T_{f}}^{i T_{s}+T_{\text {int }}} r^{2}(t) d t, \quad j=1,2, \ldots, N_{f}
$$

It is well known that the integrator output $y_{i, j}$ can be viewed as a sum of $2 M=2 W T_{\mathrm{int}}+1$ independent variables, where $W$ is the signal bandwidth. According to the Central Limit Theorem, $y_{i, j}$ can be well approximated as Gaussian random variable when $2 M$ is large enough. Let the PDF (probability density function) of $y_{i, j}$ for hypotheses $H_{0}$ and $H_{1}$ be given by $p_{0}\left(y_{i, j}\right) \sim \mathcal{N}\left(\mu_{0}, \sigma_{0}^{2}\right)$ and $p_{1}\left(y_{i, j}\right) \sim \mathcal{N}\left(\mu_{1}, \sigma_{1}^{2}\right)$, respectively, where $\mathcal{N}(\cdot, \cdot)$ denotes a Gaussian distribution. The calculation of their means and variances is straightforward and is skipped for brevity. The final result is

$$
\left\{\begin{array} { l } 
{ \mu _ { 0 } = M N _ { 0 } } \\
{ \sigma _ { 0 } ^ { 2 } = M N _ { 0 } ^ { 2 } }
\end{array} \text { and } \quad \left\{\begin{array}{l}
\mu_{1}=M N_{0}+2 \varepsilon \\
\sigma_{1}^{2}=M N_{0}^{2}+4 \varepsilon N_{0}
\end{array}\right.\right.
$$

in which, the signal energy $2 \varepsilon=\int_{0}^{T_{\text {int }}}\left(\sqrt{E_{p}} s(t)\right)^{2} d t$ is deterministic and can be estimated during a preamble step thanks to appropriate estimators [5].

\section{Conventional Energy Detector}

The conventional energy detector is the EGC detector, in which, the $N_{f}$ energy samples $y_{i, j}$, $j=1, \ldots, N_{f}$ are added to each other, with equal weights, to form the test statistic, and the decision rule is given by

$$
Y_{i} \sim \sum_{j=1}^{N_{f}} y_{i, j} \stackrel{H_{1}}{\underset{H_{0}}{<}} \eta
$$

where the decision threshold $\eta$, which minimizes the BER, can be obtained by numerical search, or evaluated using the Gaussian approximation of the test statistic $Y_{i}$. Under the assumption of equally likely information bits the average BER is given by $[1,4]$

$$
p_{e}=\frac{1}{2}\left(1-Q_{K}\left(\sqrt{\frac{4 N_{f} \varepsilon}{N_{0}}}, \sqrt{\frac{2 \eta}{N_{0}}}\right)\right)+\frac{1}{2}\left(e^{-\eta / N_{0}} \sum_{k=1}^{\left\lfloor N_{f} M\right\rfloor} \frac{\left(\eta / N_{0}\right)^{N_{f} M-k}}{\Gamma\left(N_{f} M-k+1\right)}\right)
$$

where $Q_{K}$ is the generalized Marcum-Q function of order $K=N_{f} M$ and $\Gamma(x)$ is the Gamma function.

\section{Proposed Energy Detector}

Considering (4) we note that the test statistic of EGC detector is equivalent to $\overline{y_{i}}=\frac{1}{N_{f}} \sum_{j=1}^{N_{f}} y_{i, j}$, which is an estimate of the mean of the energy samples. In fact, the EGC detector distinguishes two hypotheses by a change in the mean of the energy samples.

The proposed detector for IR-UWB is directly based on the Gaussian approximation and the identically distributed nature of $\left\{y_{i, j}\right\}_{j=1}^{N_{f}}$, the log-likelihood ratio decision rule for maximum likelihood criterion can be expressed as 


$$
L\left(y_{i, 1}, \ldots, y_{i, N_{f}}\right)=\ln \prod_{j=1}^{N_{f}} \frac{p_{1}\left(y_{i, j}\right)}{p_{0}\left(y_{i, j}\right)}=\sum_{j=1}^{N_{f}} \ln \frac{p_{1}\left(y_{i, j}\right)}{p_{0}\left(y_{i, j}\right)} \underset{H_{0}}{\stackrel{H_{1}}{\gtrless}} 0
$$

Next, substituting (3) into (6), and incorporating the non-data-dependent term into the threshold, the suboptimal decision rule can be obtained as

$$
\frac{1}{N_{f}} \sum\left(y_{i, j}-\bar{y}_{i}\right)^{2}+\bar{y}_{i}^{2}-M N \overline{y_{i}} \underset{H_{0}}{\stackrel{H_{1}}{\gtrless}} M N \varepsilon+M N^{2}\left(\frac{M N}{4 \varepsilon}+1\right) \ln \left(1+\frac{4 \varepsilon}{M N}\right)
$$

in which, $\frac{1}{N_{f}} \sum\left(y_{i, j}-\overline{y_{i}}\right)^{2}$ is just an estimation of the variance of the energy samples. This implies that the symbol decision strategy of the proposed detector relies not only on the change in mean but also on the change in variance of the energy samples.

To find the probability of error, we can rewrite (7) in the following form

$$
Y_{i}^{\prime}=\sum_{j=1}^{N_{f}}\left(y_{i, j}-\frac{M N}{2}\right)^{2} \stackrel{H_{1}}{\underset{H_{0}}{\gtrless}} \eta^{\prime}
$$

where $\eta^{\prime}=N_{f} M N^{2}\left(\frac{\varepsilon}{N}+\ln \left(1+\frac{4 \varepsilon}{M N}\right)\right)\left(\frac{M N+4 \varepsilon}{4 \varepsilon}\right)$. It is apparent form (8) that the PDF of $Y_{i}^{\prime}$ conditioned on $d_{i}=0$ (or, $d_{i}=1$ ) follows a non-central chi-square distribution with $N_{f}$ degrees of freedom and non-centrality parameter $0.5 M N \sqrt{N_{f}}$ (or, $0.5 M N \sqrt{N_{f}}+2 \varepsilon \sqrt{N_{f}}$ ). Then the average BER can be calculated as

$$
p_{e}=\underbrace{\frac{1}{2}\left(1-Q_{K}\left(\frac{1}{2} \sqrt{N_{f}\left(M+4 \frac{\varepsilon}{N}\right)}, \sqrt{\left.\frac{\eta^{\prime}}{M N^{2}+4 \varepsilon N}\right)}\right)\right.}_{p(0 \mid 1)}+\underbrace{\frac{1}{2} Q_{K}\left(\frac{1}{2} \sqrt{N_{f} M}, \sqrt{\frac{\eta^{\prime}}{M N^{2}}}\right)}_{p(10)}
$$

where $K=N_{f} / 2$, and $p(0 \mid 1), p(1 \mid 0)$ denote the BER exepressions for bits 1 and 0 , respectively.

\section{Numerical Results and Discussion}

To validate the Gaussian approximation, we compare the theoretical PDF with the simulated PDF of decision variables $Y_{i}^{\prime}$. In this example, we consider IEEE 802.15.4a CM2 channel model, $N_{0}=1$, $T_{\text {int }}=60 \mathrm{~ns}$ and $E_{p} / N_{0}=10 \mathrm{~dB}$. This is illustrated on Fig. 1, where realizations fit very well with the theoretical PDF (which is based on the Gaussian approximation).

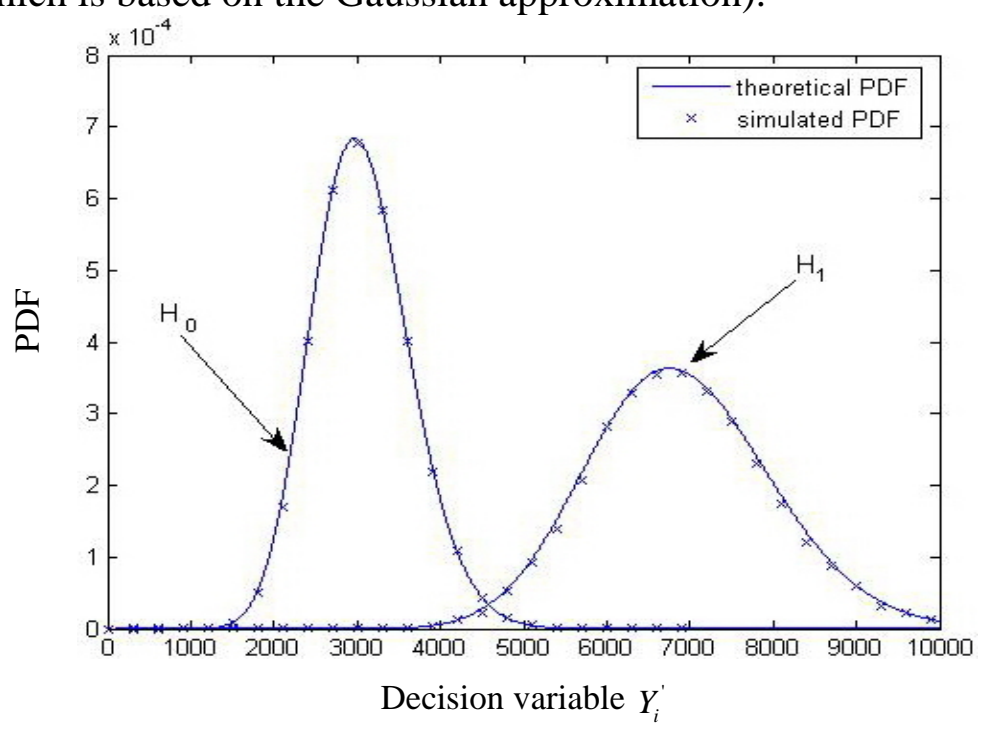


Fig. 1. PDF of the decision variable $Y_{i}{ }^{\prime}$ under hypothesis $H_{0}$ and $H_{1}$

Fig. 2 shows a comparison of the BER performances of the conventional detector with the proposed detector according to SNR $E_{p} / N_{0}$. In this simulation, the integration time $T_{\text {int }}=60 n s$ and the signal bandwidth $W=1.5 \mathrm{GHz}$ have been chosen. The estimation of the $\varepsilon$ is performed with a training sequence of 128 symbols. Fig. 2 indicates that the BER performance of the proposed detector always surpasses that of the conventional detector. Moreover, we observe that the performance improvement of proposed detector becomes more significant as $N_{f}$ becomes larger since more pulses lead to more accurate variances estimation.

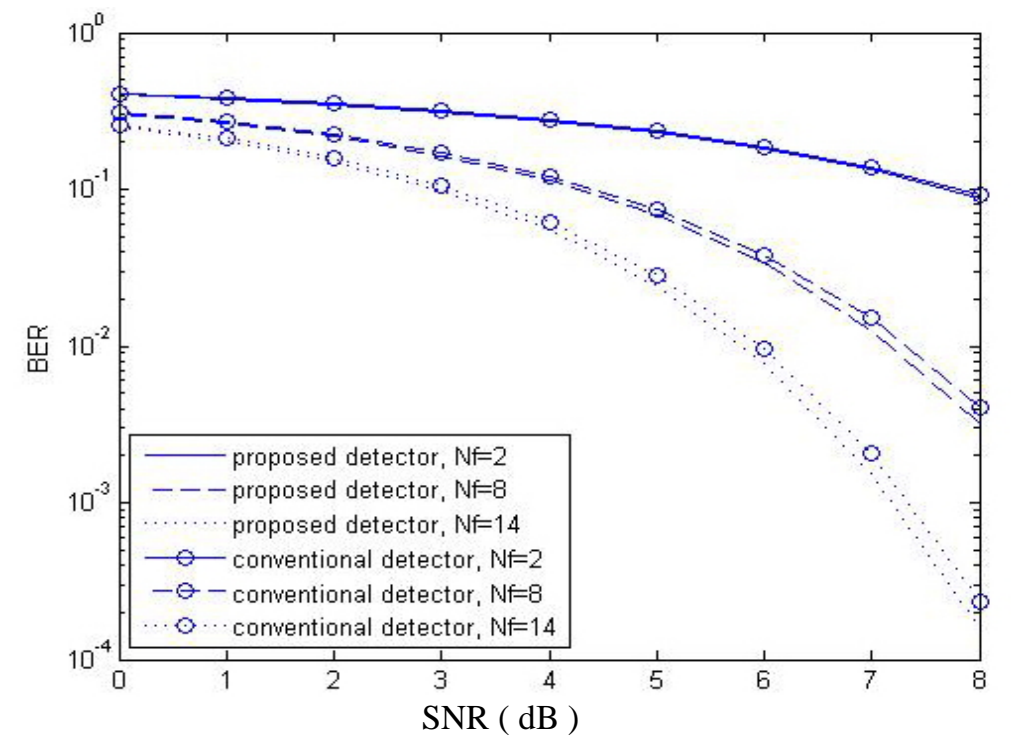

Fig. 2. BER performance comparison under IEEE 802.15.4a CM2 channel

\section{Summary}

In this paper, we have proposed a novel energy detection scheme for non-coherent IR-UWB systems. It exploits variance estimation of energy samples per symbol and approximates the optimum strategy to minimize the error probability. The proposed detector is simply a threshold detector as the conventional detector, except with the extra complexity of mapping the received signal with a quadratic function.

\section{Acknowledgements}

This work was supported by BIGC Key Project (No. E-a-2014-09) and the Importation and Development of High-Caliber Talents Project of Beijing Municipal Institutions (No. CIT\& TCD 20130329) and the Scientific Research Common Program of Beijing Municipal Commission of Education (No.KMKM201410015006).

\section{References}

[1] Wang, F., Tian Z., and Sadler Brain M., "Weighted energy detection for noncoherent ultra-wideband receiver design”, IEEE Transaction on Wireless Communication, Feb 2011, Vol, 10(2) pp. 710-720

[2] Cheng, X.T., Guan, Y. L., and Gong, Y. "Thresholdless energy detection for ultra-wideband block-coded OOK signals”, Electronics Letters, vol. 44, No. 12, 2008, pp. 755-756

[3] Gezici, S., Sahinoglu, Z. Poor, H. V.: 'On the optimality of equal gain combining for energy detection of unknown signals’, IEEE Communication Letters, vol. 10, 2006, pp. 772-774. 
[4] Paquelet, S., Aubert, L. M. and Uguen, B. “An impulse radio asynchronous transceiver for high data rates”, Proc. IEEE Ultrawideband Syst. Technol. (UWBST), Kyoto, Japan, May 2004, pp. 1-5.

[5] Paquelet, S., Aubert, L. M. : “An energy adaptive demodulation for high data rates with impulse radio”, Proc. IEEE Radio and Wireless Conf., Atlanta, USA, Sept. 2004, pp. 323-326 